\title{
Nossas diretrizes em hipertensão pulmonar
}

Romberg, ${ }^{(1)}$ em 1891, publicou a primeira descrição de hipertensão arterial pulmonar (HAP) com dados da necropsia de um paciente com cardiomegalia e hipertrofia de ventrículo direito, classificando-a como esclerose da circulação pulmonar. Wood, (2) em 1950, em uma série de 233 casos suspeitados de cardiopatia congênita, 152 dos quais submetidos a cateterismo cardíaco, definiu seis deles como de hipertensão pulmonar idiopática. Logo a seguir, Dresdale et al.(3) relataram três casos de mulheres jovens com dispnéia grave aos esforços, eletrocardiograma compatível com hipertrofia de ventrículo direito, radiograma de tórax com dilatação do tronco da artéria pulmonar e acentuada elevação das pressões da artéria pulmonar observada através de cateterismo do coração direito, de etiologia inexplicada. Estes autores consideraram tais pacientes como portadoras de uma forma primária de hipertensão pulmonar, na qual o aumento da resistência vascular pré-capilar produziria hipertrofia do coração direito, à semelhança da hipertrofia ventricular esquerda causada pela hipertensão arterial sistêmica. Assim começou a história da hipertensão arterial pulmonar, cujo impacto e interesse médico se fez sentir a partir do surto de casos decorrentes do uso do anorexígeno aminorex, na Europa central, no final da década de 1960.

Em 1973, a Organização Mundial da Saúde(4) reuniu um grupo de trabalho para discutir o problema da HAP, com recomendações que permitiriam, a seguir, uma abordagem mais sistematizada do tema. Em 1981, o National Heart, Lung and Blood Institute of Health, nos EUA, criou o Registro Nacional de pacientes com hipertensão pulmonar primária, cujos primeiros resultados foram apresentados em 1987. ${ }^{(5)} 0$ segundo encontro patrocinado pela Organização Mundial da Saúde, em $1998,{ }^{(6)}$ e um grande simpósio internacional em $2003^{(7)}$ deram a devida dimensão ao problema e firmaram a definitiva institucionalização da hipertensão arterial pulmonar como uma realidade clínica.

A possibilidade de se observar a circulação pulmonar central pelo ecodopplercardiograma trouxe a hipertensão pulmonar até o consultório do pneumologista. A angiotomografia computadorizada helicoidal abriu uma janela não invasiva para quase toda a circulação pulmonar. A bibliografia internacional, sob o apoio entusiasmado da indústria farmacêutica, passou a oferecer permanentemente informações sobre a patogenia, a fisiopatologia, os novos recursos que permitiam a identificação de HAP e as possibilidades de se oferecer tratamento aos pacientes - nem tantos, é verdade que procuravam os médicos com dispnéia.

Em 1996, durante o congresso da Sociedade Brasileira de Pneumologia e Tisiologia (SBPT), em Belo Horizonte (MG), prepusemos a criação da Comissão de Circulação Pulmonar, cujos primeiros signatários foram os ilustres e saudosos professores Mario Rigatto e Octávio Ribeiro Ratto, ambos com significativa contribuição ao estudo da circulação pulmonar. Os temas mais prevalentes da circulação pulmonar tromboembolia venosa e hipertensão arterial pulmonar - passaram a receber espaço nos eventos da SBPT e a serem discutidos com acento local.

Temos agora nossas esperadas diretrizes para a HAP. 0 Dr. Rogério Souza assumiu a responsabilidade por sua coordenação, não só por ser o presidente da comissão, mas também pelo mérito de suas importantes contribuições ao tema, como atestam as publicações nacionais e internacionais de seu grupo. ${ }^{(8-10)}$ Nossa realidade não é a mesma dos centros produtores de conhecimento e com grandes investimentos em pesquisa. A tríade dispnéia/ecocardiograma com HAP/sildenafil banaliza o manejo da doença e oferece riscos aos pacientes. Novos fármacos a serem lançados no país precisam de normatização clara para o melhor custo-benefício dentro de nossa realidade social e econômica. Cumprimentos a todo seleto grupo de colaboradores pelo serviço prestado à SBPT e a seus pacientes.

Sérgio Saldanha Menna-Barreto

Prof. Titular da Faculdade de Medicina da Universidade Federal do Rio Grande do Sul 


\section{REFERÊNCIAS}

1. Rubin LJ. Primary pulmonary hypertension. ACCP Consensus Statement. Chest. 1993;104(1):236-50.

2. Wood P. Congenital heart disease. A review of its clinical aspects in the light of experience gained by means of modern techniques. Br Med J. 1950;2(4681):693-8.

3. Dresdale DT, Schultz M, Michtom RJ. Primary pulmonary hypertension. I. Clinical and haemodynamic study. Am J Med. 1951;11(6):686-705.

4. Hatano S, Strasser T. editors. Primary pulmonary hypertension: Report on a WHO Meeting. Geneve: Geneva: World Health Organization; 1975. 45p.

5. Rich S, Dantzker DR, Ayres SM, Bergofsky EH, Brundage BG, Detre KM, et al. Primary pulmonary hypertension: a national prospective study. Ann Intern Med. 1987;107(2):216-23.

6 . Rich S, editor. Primary pulmonary hipertension. Executive Summary from the World Symposium on Primary Pulmonary Hypertension 1998 [text on the Internet]. [cited 2004 Nov
20]. Evian, France, 6-10 Sep 1998. Available from: http:// www.who.int./ncd/cvd/pph.html

7. Proceedings of the 3rd World Symposium on Pulmonary Arterial Hypertension. Venice, Italy, June 23-25, 2003. J Am Coll Cardiol. 2004;43(12 Suppl S):1S-90S.

8. Souza R, Bogossian HB, Humbert M, Jardim C, Rabelo R, Amato $M B$, et al. N-terminal-pro-brain natriuretic peptide as a haemodynamic marker in idiopathic pulmonary arterial hypertension. Eur Respir J. 2005;25(3):509-13.

9. Souza R, Amato MBP, Demarzo SE, Deheinzelin D, Barbas CSV, Caruso $P$, et al. Ação da adenosina na circulação pulmonar de pacientes com hipertensão arterial pulmonar. J Bras Pneumol. 2005;31(1):20-4.

10. Souza R, Amato MBP, Demarzo SE, Deheinzelin D, Barbas CS, Schettino GP, et al. Pulmonary capillary pressure in pulmonary hypertension. Crit Care. 2005;9(2):R132-8. 